\title{
Optimisation of multiple encapsulated electrode plasma actuator
}

\author{
Rasool Erfani $^{\text {a }}$, Tohid Erfani ${ }^{b}$, Sergei V. Utyuzhnikov ${ }^{a}$, Konstantinos Kontis ${ }^{\text {a,* }}$ \\ a Aero-Physics Laboratory, School of Mechanical, Aerospace and Civil Engineering, University of Manchester, UK \\ b Civil, Environmental and Geomatic Engineering, University College London, London, UK
}

\section{A R T I C L E I N F O}

\section{Article history:}

Received 6 October 2011

Received in revised form 21 December 2011

Accepted 15 February 2012

Available online $\mathrm{xxxx}$

\section{Keywords:}

Flow control

Plasma actuator

Dielectric barrier discharge

Multiple encapsulated electrode plasma actuator

Surrogate modelling and optimisation

\begin{abstract}
A B S T R A C T
The standard dielectric barrier discharge plasma actuator, in which an asymmetric arrangement of electrodes leads to momentum coupling into the surrounding air, has already demonstrated its capability for flow control. The new design of such an actuator exploits the multi-encapsulated electrodes to produce higher velocities providing more momentum into the background air. As the number of encapsulated electrodes increases and other variables such as the driving frequency and voltage amplitude are considered, finding the optimum actuator configuration for increasing the induced velocity becomes a challenge. Specially the task is prohibitive if it is implemented on an ad hoc basis. This paper uses D-optimal design to identify a handful of experiments, for which the velocity is obtained by Particle Imaging Velocimetry measurement. Afterwards, the velocity is modelled through a surrogate modelling practice, and the model is validated both experimentally and statistically. To find the optimum actuator configuration, numerical optimisation is conducted and the results are investigated through experiment. The results show that the surrogate modelling approach provides a cheap and yet efficient method for systematically investigating the effect of different parameters on the performance of the plasma actuator.
\end{abstract}

(c) 2012 Elsevier Masson SAS. All rights reserved.

\section{Introduction and motivation}

During the past decade in the field of flow control, the use of plasma actuators has been implemented by many researchers. The new approach involves the use of a dielectric barrier discharge (DBD) that is comprised of two asymmetric electrodes separated by a dielectric material. One of the electrodes is typically exposed to the air and connected to the high voltage supply while the other electrode is fully covered by the dielectric material and earthed as shown in Fig. 1. A high potential difference with typical voltages of $2 \mathrm{kV}_{p-p}$ to $40 \mathrm{kV}_{p-p}$ (peak to peak) and frequencies of $300 \mathrm{~Hz}$ to $1 \mathrm{MHz}$ weakly ionizes the surrounding gas over the hidden electrode. Having no moving parts in actuator configuration, near instantaneous response, relatively low power consumption in addition to a wide range of operational frequencies have made this device an attractive alternative to other active flow control methods such as piezoelectric actuators, synthetic jets, and vortex generators [11]. DBDs have the ability in boundary layer manipulation $[19,8,29,25]$, separation delay on aerofoils and turbine blades $[26,18]$, manipulation of the laminar to turbulent transition point [13], control of separation on stationary [17] and oscillating aerofoils [27].

In spite of the inherent advantages (no moving parts and vast control potential) of the DBD actuator, it is essential for plasma

\footnotetext{
* Corresponding author.

E-mail address: k.kontis@manchester.ac.uk (K. Kontis).
}

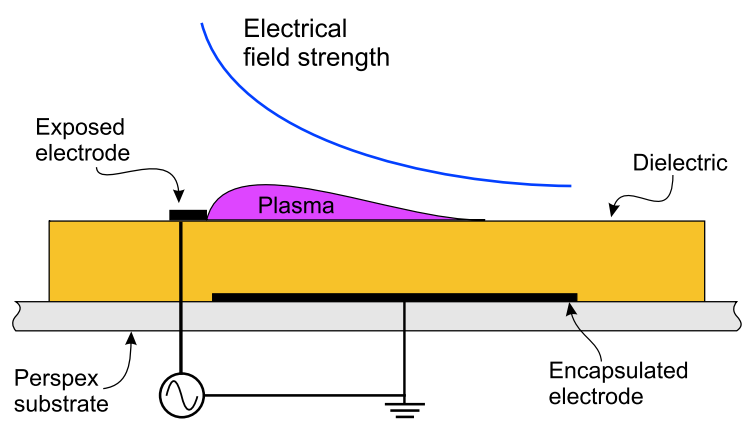

(a) Standard actuator
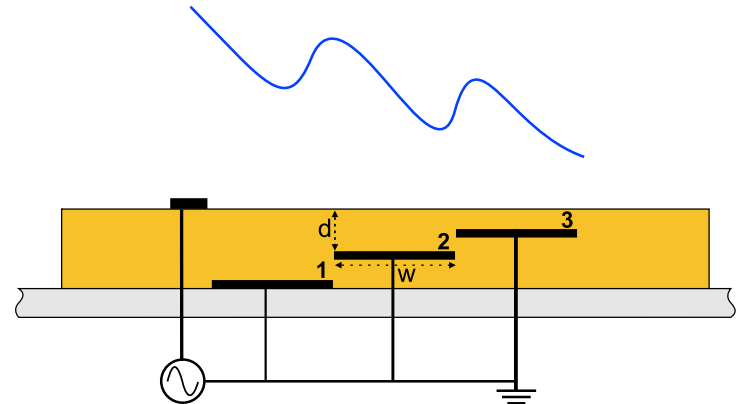

(b) Actuator with multiple encapsulated electrodes

Fig. 1. The hypothetical effect of MEE plasma actuator on electric field strength. 
actuators to produce higher induced jet velocities and impart more momentum to be applicable in higher Reynolds number flows. One way to increase the impact of an actuator on the flow is the variation of the actuator geometry. Santhanakrishnan et al. [32] introduce an annular configuration of the plasma actuator that produces a vertical jet. Further, series of linear actuators is adopted by Roth et al. [30] and Forte et al. [10] to increase the induced velocity. In addition to the geometry variation of the actuator, another way for increasing the body force and in turn the induced velocity is by increasing the number of ions $(\mathrm{Ne})$, or the strength of electric field $(E)$, or the both:

$F=N e E$

Increasing the voltage and frequency of the input signal increases the electric field strength $[9,24]$ while the input waveform varies the number of charged particles per unit of time delivering momentum to the neutral air [4]. The ion number density, the electric field, and its gradients above the actuator dielectric can also be maximised by altering the atmospheric pressure and temperature [34] and also by changing the dielectric material, width [29,9] and temperature [7].

A new design of plasma actuator developed at the University of Manchester has been shown to increase the induced velocity by 90\% using multiple encapsulated electrodes [14-16]. In the standard actuator design the electric field decays as one moves further away from the exposed electrode. In this new design, multiple encapsulated electrode (MEE) is used to produce a variable electric field for accelerating the charged particles to higher velocities. This provides more momentum to the neutral background air (Fig. 1).

In the proposed study, the effect of one parameter on velocity is being studied while the others are kept constant at a prescribed value. This process continues for all the parameters until a relationship between velocity and actuator configuration is found. Although this methodology provides the best configuration amongst the implemented experiments, there is no guarantee for finding the best possible configuration. In addition, the final configuration is limited to the already implemented setups which may not be optimum globally. It also should be taken into account that each experiment is an expensive job to be undertaken with the possibility of many redundant and unused experiments leading to unwanted and unnecessary expenses. Furthermore, the proposed approach is an exhaustive process even if the number of problem parameters is small. Added to this difficulty is the investigation of the true value of velocity in the presence of human uncertainty and error in recording the observations. Therefore, a more systematic approach for finding a relationship among the parameters and the induced velocity should be pursued.

Surrogate modelling alleviates the above burdens by constructing approximation models. These models effectively follow the behaviour of the simulation results as closely as possible. The methodology is able to set the most informative set of experiments while keeping the number of experiments as low as possible. As each experiment is costly to be implemented and time consuming to be observed, the latter property is important to be recognized.

Typically, surrogate modelling includes response surface methodology (RSM) [21], neural networks [1] evolutionary programming [22], and kriging interpolation [12]. Examples of widely used surrogate modelling include the multidisciplinary design of the high speed civil transport [35], structural optimisation [20], blade shape design optimisation [31] and aerospike nozzle design [33]. In plasma actuator study [3], used four surrogate models to investigate the parametric effects of the DBD actuator in helium by simulating a 2-species fluid model. They investigate the effect of waveform, frequency of the applied voltage and dielectric constant on both the power input and generated thrust of the DBD actuators.
This paper employs the surrogate modelling technique for maximisation of the induced velocity as a function of voltage amplitude (vol), driving frequency (freq), and geometries of three insulated electrodes. The experiments have been implemented in the ambient air. A numerical optimisation is carried out to find the optimum configuration for maximisation of the velocity.

The rest of the paper is organized as follows. Section 2 gives the overview of the problem and optimisation formulation. In Section 3, design of experiments and the modelling issues are illustrated. In addition, the setups are explained and experiments are conducted. Section 4 deals with the velocity model approximation and its validation. The model is optimised in Section 5 and the results are presented and discussed. The paper is concluded in Section 6 where some recommendation for future work is given.

\section{Problem definition}

As mentioned earlier, the purpose of this paper is to present a systematic methodology in order to find the best possible configuration of plasma actuator to achieve the maximum induced velocity. The new configuration of plasma actuator uses multiple encapsulated electrodes. Previously [14], we have noticed that using multiple encapsulated electrodes affects the induced velocity substantially. Therefore, we choose this actuator for our optimisation task. Indeed, we systematically study the possibility of further improvement in induced velocity by any possible different configuration than we have already tested via the experiments presented in Hale et al. [14]. A sketch of the electrodes is in Fig. 1. A plasma actuator consists of one exposed electrode and three more electrodes positioned under it and isolated by a dielectric material, in this case, Kapton. In Fig. 1, the geometry of the three electrodes are taken as the model parameters. Finding the best possible configuration by changing these parameters as well as the power supply characteristics is an exhaustive and expensive job. Optimisation methodology can be employed to address this issue. To realize this, the above problem may be formulated as the following optimisation task:

\section{Maximise velocity}

subject to: $180 \leqslant d_{1}, d_{2}, d_{3} \leqslant 540 \mu \mathrm{m}$,

$$
\begin{aligned}
& 5 \leqslant w_{1}, w_{1}, w_{3} \leqslant 40 \mathrm{~mm}, \\
& 10 \leqslant \text { vol } \leqslant 16 \mathrm{kV}_{p-p}, \\
& 1 \leqslant \text { freq } \leqslant 20 \mathrm{kHz},
\end{aligned}
$$

where $d_{i}$ and $w_{i}, i=1,2,3$, are the depth of the electrodes from the surface and the electrode width, respectively (see Fig. 1). In addition, vol and freq are the voltage amplitude and the deriving frequency that can be varied by the power supply. The design variables are listed in Table 1 . It should be noted that, following the ranges presented in Hale et al. [14,15] the same intervals for the design variables are chosen to make the future comparisons possible. For velocity estimation in problem (2), Response Surface Methodology (RSM) is implemented next. One may note that right after velocity estimation and model validation, the velocity equation will be exploited for the optimisation of Eq. (2).

\section{Modelling and experimental setup}

\subsection{Velocity approximation}

RSM is an approximation technique which performs a series of experiments for a predefined set of design points (independent variables). Using the responses (observations) obtained, RSM constructs a surface over the design space. For obtaining such 
Table 1

Design variables ranges.

\begin{tabular}{|c|c|c|c|c|c|c|c|}
\hline Name & $\operatorname{vol}\left(\mathrm{kV}_{p-p}\right)$ & freq $(\mathrm{kHz})$ & $d_{1}(\mu \mathrm{m})$ & $d_{2}(\mu \mathrm{m})$ & $d_{3}(\mu \mathrm{m})$ & $w_{1}(\mathrm{~mm})$ & $w_{3}(\mathrm{~mm})$ \\
\hline Level & $\{8,10,12,14,16\}$ & $\{1,5,7,10,20\}$ & $\{180,360,540\}$ & $\{180,360,540\}$ & $\{180,360,540\}$ & $\{5,10,20,40\}$ & $\{5,10,20,40\}$ \\
\hline
\end{tabular}

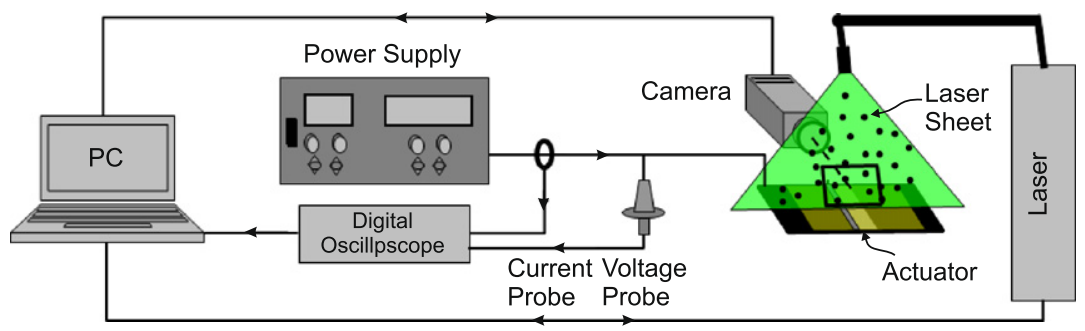

Fig. 2. Schematic of PIV experimental setup.

a surface, RSM implements regression analysis for the following quadratic function

$$
F=\text { velocity }=b_{0}+\sum_{j} b_{j} x_{j}+\sum_{j} \sum_{i} b_{i j} x_{i} x_{j}
$$

where $F$ is the response function (velocity of the induced jet), $x_{i}$ and $x_{j}$ are the design variables (Table 1 ), and $b_{0}, b_{j}, b_{i j}$ are the unknown polynomial coefficients. The reason to choose Eq. (3) as a function of the order of two is due to the following facts. The cubic and higher order polynomials need large number of experiments which makes the RSM an expensive task. In addition, cubic and higher order polynomials generate the local optimum in the response surface. This abandons some optimisers to obtain the global optimum point in an optimisation process.

To approximate the velocity, a set of the most informative experiments is required. Design of Experiments (DoE) [2] is a set of methods for selecting an appropriate sample of experiments efficiently and includes full and fractional Factorial designs, PlackettBurman designs, Central-Composite designs, Taguchi designs and D-optimal designs [21]. Among the alternatives, D-optimal has a favorable properties. It requires a fewer number of experimental simulations compared to the other methodologies [21]. Furthermore, if there exists a constraint in the problem, D-optimal can efficiently sample an appropriate set of experiments. The latter property is required for our purpose. This is because the thickness of the dielectric, the distance of the electrodes from the surface, the total width of the plasma actuator and limited ranges of voltage and frequency constrain the design space. This includes that not all the electrodes can be positioned on the same level except for the deepest level. Further, due to laboratory resource constraints, the combination of extreme voltage and frequency, $16 \mathrm{kV}_{p-p}$ and $20 \mathrm{kHz}$, is not viable. This implies that not all the configuration setups are experimentally feasible to be investigated. D-optimal can be implemented in such a design space to sample the experiments properly.

The criterion in D-optimal design is to select a set of experiments for which the variance (uncertainty) of the estimated coefficients of the polynomial model (3) is minimised. This is equivalent to maximising the determinant of the information matrix [21] (see Appendix A). The ranges of each design variable can be found in Table 1 from which different experiment setups are picked out. In fact, these values are the ones which can be tested with the equipments available in our laboratory. In addition, $w_{2}$ is not present in Table 1 as is justified next.

\subsection{Experimental setup and results}

In Eq. (3), we consider all the linear terms except for $w_{2}$. This is illustrated as follows. Following the paper by Enloe et al. [5] and Forte et al. [10], actuator with wider encapsulated electrode(s) can expand plasma farther. However, plasma cannot expand so far on the plane even with a wider electrode. In our previous experiment [15], it has been concluded that having encapsulated electrode more than $50 \mathrm{~mm}$ does not increase the induced velocity. Therefore, the total actuator width is set to $50 \mathrm{~mm}$. As a result, having set the $w_{1}$ and $w_{3}$, we are able to simply determine the $w_{2}$. In addition, all the quadratic and interaction terms are not included in velocity approximation. This is due to our experimentally screening step. It is also recognized that not all the interactions are significant or even necessary to be investigated [15]. Thus, in the numerical model, we only choose those terms deem to be experimentally effective and important to be examined. Therefore, in total there are 16 coefficients in Eq. (3) to be estimated including seven linear, five quadratic (frequency, voltage and depth of the electrodes) and four interaction terms ( $\mathrm{vol} \times d_{1}$, vol $\times w_{1}, d_{1} \times w_{1}$ and $d_{3} \times w_{3}$ ).

Using the information in Table 1 and depending on our resource limitations and configuration constraints explained earlier, we conduct 25 experiments with different plasma configurations using D-optimal approach implemented in Matlab. It should be noted that using Full Factorial design as an alternative to the D-optimal one, it would be needed for a total of $5^{2} \times 3^{3} \times 4^{2}=10,800$ test setups. Obviously this requires a large number of experimental runs leading to a high computational expenses.

The velocity fields generated are recorded using Particle Imaging Velocimetry (PIV) [28]. PIV basically uses the displacement of particles to determine various flow field parameters. A laser is manipulated into a laser sheet using lenses and is used to illuminate the region of interest. Using two successive images, separated by a known time, statistical analysis can be performed to measure the instantaneous velocity. In this paper, the PIV measurements are performed using an NDYAG 532/1064 nm, Litron $200 \mathrm{~mJ}$, pulsed laser as the light source. The laser pulses with a repetition rate of $15 \mathrm{~Hz}$, and a pulse width of $4 \mathrm{~ns}$. In the performed experiments, the duration between pulses is set at $400 \mu$ s based on the field of view size and expected induced velocity, of the order of $4 \mathrm{~m} / \mathrm{s}$. The experimental setup is presented in Fig. 2. The laser is delivered using a laser arm mounted above the actuator to produce a laser sheet that run along the centre-line of the actuator span, normal to the electrodes. To improve PIV measurements near the surface the following actions are implemented: firstly the surface is painted mat black, secondly by using a laser power that is low enough to obtain a good signal from the particles but at the same time leads to minimum surface reflections. The actuator is further located in a sealed chamber and seeded using a 6 jet atomizer that produced light-scattering olive oil particles with a size of approximately $1 \mu \mathrm{m}$. 
Table 2

Sample data for D-optimal design.

\begin{tabular}{|c|c|c|c|c|c|c|c|}
\hline \multicolumn{7}{|c|}{ Design variables } & \multirow{2}{*}{$\frac{\text { Response }}{\text { Velocity }}$} \\
\hline vol & freq & $d_{1}$ & $d_{2}$ & $d_{3}$ & $w_{1}$ & $w_{3}$ & \\
\hline 16 & 10 & 540 & 540 & 540 & 40 & 5 & 1.70 \\
\hline 16 & 10 & 540 & 540 & 360 & 20 & 10 & 2.28 \\
\hline 8 & 10 & 180 & 180 & 540 & 5 & 40 & 1.20 \\
\hline 16 & 10 & 540 & 360 & 180 & 40 & 5 & 2.32 \\
\hline 8 & 10 & 540 & 540 & 180 & 5 & 40 & 0.31 \\
\hline 12 & 10 & 180 & 360 & 540 & 5 & 40 & 1.44 \\
\hline 8 & 10 & 540 & 180 & 180 & 40 & 5 & 0.55 \\
\hline 16 & 10 & 540 & 540 & 180 & 5 & 40 & 1.91 \\
\hline 12 & 20 & 180 & 360 & 540 & 40 & 5 & 1.27 \\
\hline 12 & 1 & 360 & 360 & 540 & 5 & 40 & 0.82 \\
\hline 12 & 20 & 180 & 540 & 540 & 10 & 20 & 1.58 \\
\hline 12 & 10 & 180 & 540 & 540 & 10 & 20 & 2.13 \\
\hline 12 & 20 & 540 & 540 & 540 & 5 & 40 & 0.79 \\
\hline 16 & 10 & 540 & 180 & 180 & 10 & 20 & 1.87 \\
\hline 14 & 10 & 180 & 360 & 540 & 5 & 40 & 1.71 \\
\hline 14 & 10 & 180 & 180 & 540 & 20 & 10 & 1.68 \\
\hline 16 & 10 & 540 & 180 & 180 & 40 & 5 & 2.43 \\
\hline 12 & 1 & 540 & 180 & 180 & 10 & 20 & 0.39 \\
\hline 12 & 1 & 540 & 360 & 360 & 40 & 5 & 0.70 \\
\hline 8 & 10 & 540 & 540 & 540 & 40 & 5 & 0.49 \\
\hline 12 & 1 & 180 & 540 & 540 & 40 & 5 & 0.52 \\
\hline 16 & 10 & 540 & 360 & 180 & 5 & 40 & 2.01 \\
\hline 8 & 10 & 180 & 360 & 540 & 40 & 5 & 0.92 \\
\hline 12 & 20 & 360 & 540 & 540 & 40 & 5 & 1.10 \\
\hline 12 & 10 & 540 & 540 & 180 & 20 & 10 & 1.37 \\
\hline
\end{tabular}

Table 2 summarizes the value of velocity measured for each setting of the sample data. Using this value, regression analysis [21] is implemented to fit a model for the velocity based on the explained terms of Eq. (3). In regression analysis, $b_{i}$ from Eq. (3) are estimated by minimising the error in the approximation at the sampled design points in a least square sense. Next, we numerically and experimentally examine the velocity model.

\section{Model validation}

In the subsequent subsections, we validate the model accuracy. To realize this, statistically, some measures for goodness of fitness are employed whereas in the experimental part, the model is validated by predicting the experimental results of our past studies $[15,16]$.

\subsection{Statistical model assessment}

To ensure the accuracy of the fitted model, the goodness of fit analysis is performed [21]. The approximation model is evaluated based upon different statistic measurements namely, the coefficient of determination statistic $\left(R^{2}\right)$, adjusted $R$-squared statistic $\left(\operatorname{adj} R^{2}\right)$, root mean square error (RMSE) and the regression significance $p$-value. The first two statistics denote the proportion of the variability in the sampled response that is accounted for by the approximate model. Therefore, a value above e.g. 80 percent for the first two may indicates a rational results. RMSE is an estimated error of the approximation obtained from the sample points. Thus, a lower value is preferable. $p$-value is an indication of whether the obtained observation is significant or not. $p$-value below a significance level of 0.05 is considered as statistically significant result. Based on these measurements shown in Table 3, the model is statistically approved.

For further investigations, error analysis is conducted, where error is defined by the difference between the real response (velocity) and the approximated value. The results imply that at most the error is about 0.3 while on average, the relative error in approximate model is about 9 percent. In addition, the residuals plots show that the errors are normally distributed for the surrogate
Table 3

Goodness of fit statistic

\begin{tabular}{lll}
\hline$R^{2}$ & $\operatorname{adj} R^{2}$ & RMSE \\
$95.61 \%$ & $86.80 \%$ & 0.13 \\
\hline$p$-value & Max |error $\mid$ & Mean $\mid \%$ error $\mid$ \\
0.002 & 0.27 & 9.01 \\
\hline
\end{tabular}
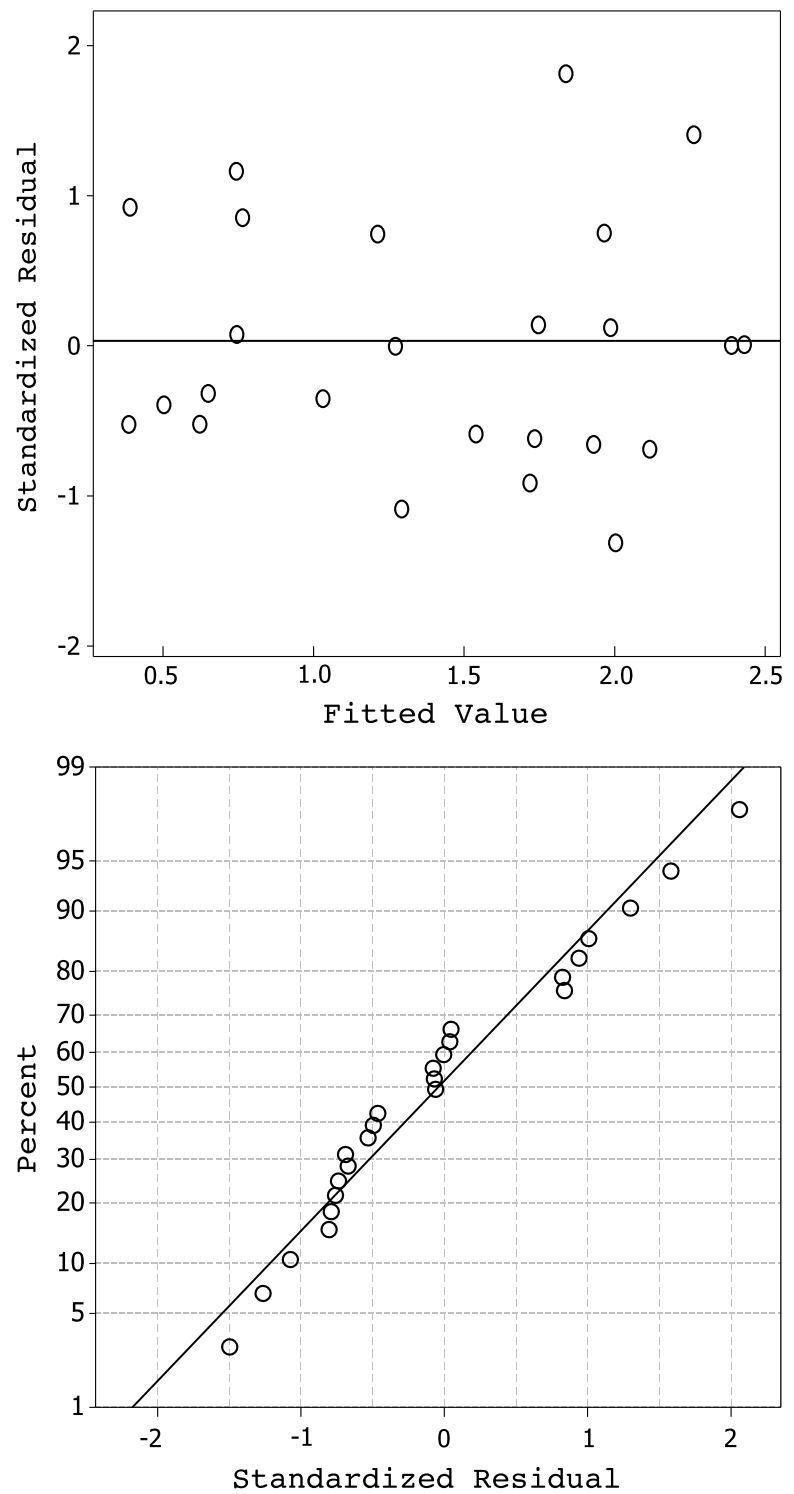

Fig. 3. Fitted values versus residual (top) and normal plot of residuals (bottom).

model (Fig. 3). All in all, the above facts and figures suggest that the model is a reasonable fit to the experimental data.

Next, we compare the numerical derived approximate model for velocity of this study with those of already experimented and presented by Hale et al. $[14,15]$ to further validate the numerical model with experimental results.

\subsection{Experimental model assessment}

It is necessary to assess the fidelity of the numerical result. In order to experimentally validate the proposed model, various actuators examined by Hale et al. [15,16] are used. Fig. 4 illustrates a schematic of the actuators. All the electrodes are tinned copper foils, $74 \mu \mathrm{m}$ thick and $100 \mathrm{~mm}$ in length, in the spanwise direction. Layered Kapton tape is used as a dielectric material with each layer 


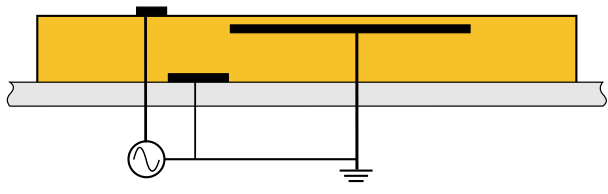

(a) Case 1

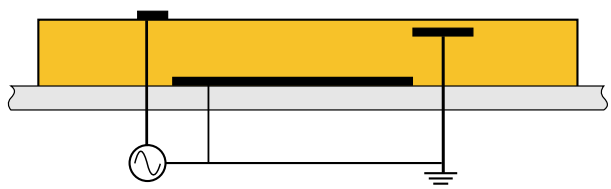

(b) Case 2

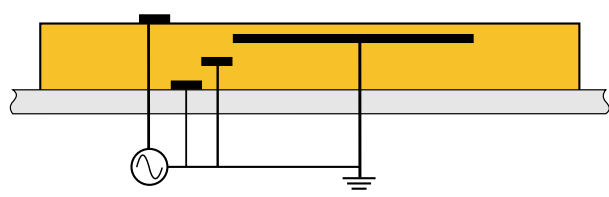

(c) Case 3

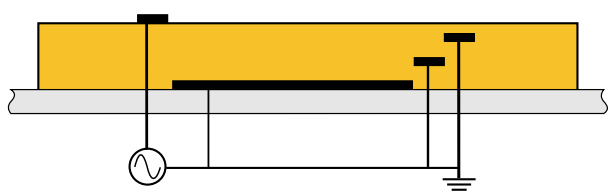

(d) Case 4

Fig. 4. Schematic of the various actuators evaluated.

Table 4

Different MEE-SDBD configurations.

\begin{tabular}{lllllll}
\hline Case & $d_{1}(\mu \mathrm{m})$ & $w_{1}(\mathrm{~mm})$ & $d_{2}(\mu \mathrm{m})$ & $w_{2}(\mathrm{~mm})$ & $d_{3}(\mu \mathrm{m})$ & $w_{3}(\mathrm{~mm})$ \\
\hline 1 & 540 & 10 & - & - & 180 & 40 \\
2 & 540 & 40 & - & - & 180 & 10 \\
3 & 540 & 5 & 360 & 5 & 180 & 40 \\
4 & 540 & 40 & 360 & 10 & 180 & 5 \\
\hline
\end{tabular}

having a $60 \mu \mathrm{m}$ thickness. The actuators are mounted on a Perspex substrate and there is no offset between the edges of successive electrodes.

The description of different configurations of plasma actuator is given in Table 4 based on the positioning of encapsulated electrodes inside the dielectric material. The exposed electrode is $5 \mathrm{~mm}$ width mounted on top of the surface.

Experimental data of induced velocity magnitude, as depicted in Fig. 5, is based on voltage variation with constant frequency of $10 \mathrm{kHz}$ while experimental data represented in Fig. 6 corresponds to frequency variation while voltage is kept constant at $12 \mathrm{kV}_{p-p}$. The presented numerical model shows a good matching with experimental results. The numerical trend coincides with individual experimental data at some points both qualitatively and quantitatively (see Figs. 6 and 5). This demonstrates that the prediction of induced velocity by the current model is reasonable.

\section{Optimal configuration setting}

Validation procedure suggests that the velocity model originated from Eq. (3) is capable for being used for further optimisation. For optimisation, sequential quadratic programming [23] is implemented as the optimiser via Matlab programming. Having optimised the velocity over the constraints introduced in Eq. (2) and presented in Table 1, the optimum results are tabulated in $\mathrm{Ta}$ ble 5 . It should be noted that $w_{2}$ is set to 40 based on the results

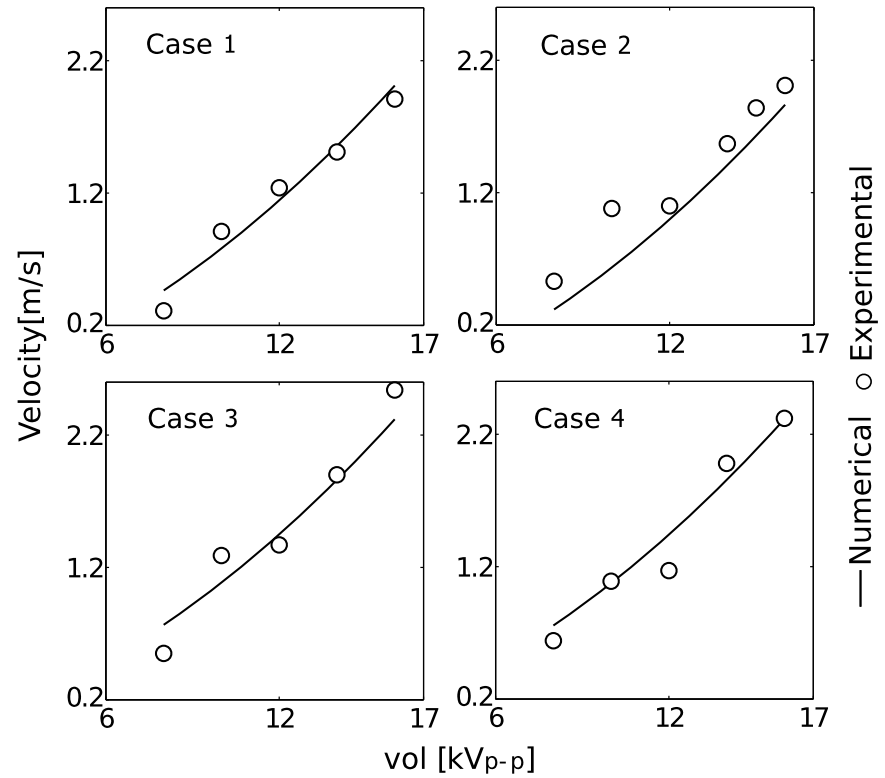

Fig. 5. Comparison between numerical and experimental results, voltage variation.

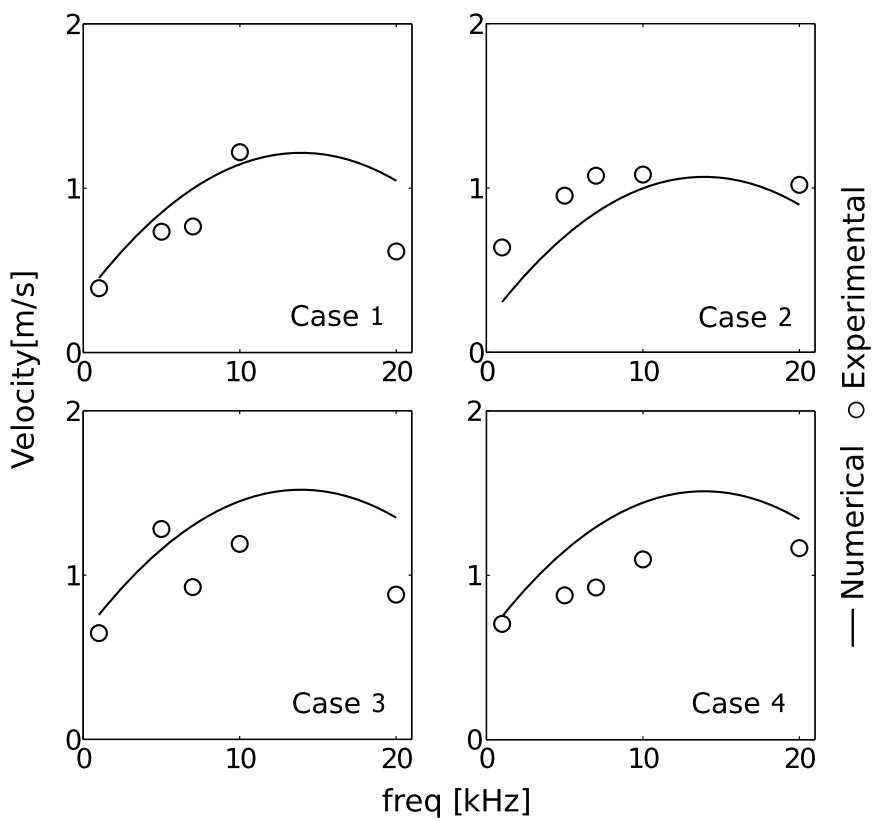

Fig. 6. Comparison between numerical and experimental results, frequency variation.

Table 5

Final configuration setting.

\begin{tabular}{|c|c|c|c|c|c|c|c|}
\hline \multicolumn{7}{|c|}{ Optimum setting } & \multirow{2}{*}{$\frac{\text { Optimum response }}{\text { Predicted velocity }}$} \\
\hline vol & freq & $d_{1}$ & $d_{2}$ & $d_{3}$ & $w_{1}$ & $\overline{w_{3}}$ & \\
\hline 16 & 14 & 245 & 470 & 210 & 5 & 5 & 2.9 \\
\hline
\end{tabular}

of $w_{1}$ and $w_{3}$ and the required total width of plasma actuator (50 mm).

Contour plots of velocity with respect to some of the most influential design variables are presented in each graph of Fig. 7 while the other variables are kept at their optimal values. These variables are chosen for illustration based on their effect on the velocity model justified by experimental experience. Fig. 7(a) illustrates that increasing voltage and frequency lead to higher velocities. Setting constant input driving frequency and increasing 

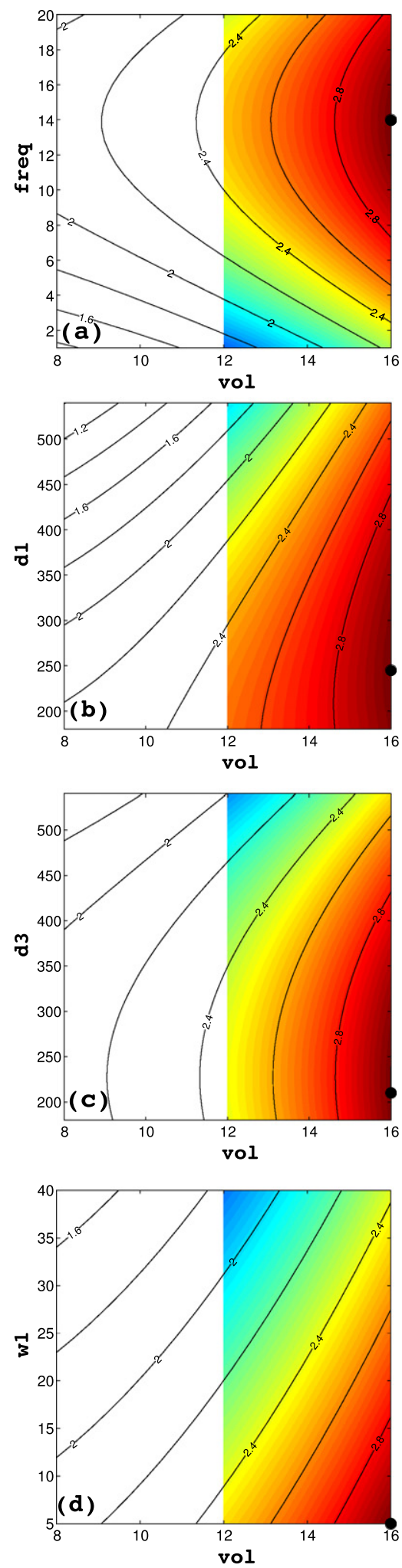

Fig. 7. Velocity contour plot with respect to the design variables (black circle indicates the optimum point).

voltage has a positive influence on the induced velocity of the jet and increases its magnitude. Also, for a certain voltage, increasing frequency causes the actuator to induce higher velocities. However, a threshold can be seen in frequency values up to $14 \mathrm{kHz}$. The capability of plasma actuator for inducing maximum velocities highly depends on the depth of the first and last encapsulated electrodes.

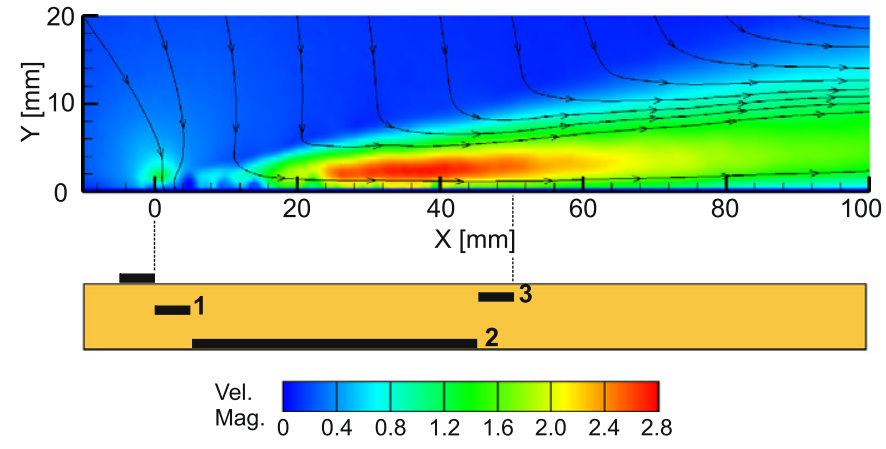

Fig. 8. PIV measurement of the flow field caused by optimum plasma actuator working with optimum input signal.

Fig. 7(b) depicts that by increasing input voltage, in order to maintain the same magnitude for the induced velocity, the distance of the first electrode from the surface should increase. It can be implied from Fig. 7(b) and (c) that for higher voltages, in order to get the higher velocity, the depth of the last hidden electrode should be less or equal than that of the first electrode whilst in lower voltages the reverse relationship holds. The velocity magnitude of the induced jet is also sensitive to the width of the first encapsulated electrode. As Fig. 7(d) presents, to reach higher velocities, $w_{1}$ should be less than $30 \%$ of the total length of encapsulated electrodes, $50 \mathrm{~mm}$.

The aforementioned numerical investigation from the simulation is consistent with the experimental evidences. It is examined experimentally that for having actuator worked in higher input powers, higher voltage amplitudes and driving frequencies, the depth of the first electrode should be sufficiently high. The initial electrode being closer to the surface leads to the formation of filaments in higher voltages and frequencies which result in the failure of the dielectric barrier [14]. However, for the sufficient plasma extension, it is required that the first electrode be placed closer to the surface in lower voltages.

Moreover, increasing the applied frequency or voltage to extremes results in the formation of coarse filaments that coexist with the filamentary plasma. The coarse filaments appear as bright streamers distributed randomly along the span of the actuator Presence of filaments is in accordance with the reduction in induced recorded velocity which is a possible reason for observing threshold at $14 \mathrm{kHz}$ in Fig. 7(a).

Fig. 8 illustrates the flow field on top of the actuator with optimum position and dimensions of encapsulated electrodes buried inside the dielectric, supplying with the optimum voltage and frequency, according to Table 5 . The maximum velocity magnitude captured by the proposed actuator working with optimum applied sine wave signal is $2.82 \pm 0.1 \mathrm{~m} / \mathrm{s}$. Considering the error analysis in Table 3, the experimental and numerical maximum velocities are accordant.

\section{Conclusion and future work}

In this paper, we propose a systematic approach to study the velocity of the airflow caused by the MEE plasma actuator. Response surface methodology (RSM) is exploited for this purpose. The D-optimal experimental design is used to keep the dimensionality of the design space, while minimising the number of experiments. After fitting the data to a quadratic polynomial, the model is validated by experimental evidence while it is shown that the model is also statistically significant. The value of the velocity is then optimised subject to the problem constraints via an optimisation algorithm. The results show promising performance and the detailed analysis helps to understand the problem dependencies. 
As the scale of the parameters suggests, this paper presents the macroscopic analysis which may bring some limitations to the approach. It is well known that the ability of the statistical technique for model estimation varies depending on experimental conditions, the noise level and error in calibration which need to be set well in order to avoid the complexity for both velocity estimation and optimisation procedure. Nevertheless, the current work is a general framework for studying the velocity of the induced jet produced by MEE plasma actuator and can be directly implemented for other setup configurations. For further investigation, it is worth looking simultaneously at the velocity and the power consumption. This leads to multiobjective optimisation and finding the best tradeoff for the actuator performance characteristics [6]. This allows the researcher to find the best possible configuration in order to maximise the airflow meanwhile keeping the power minimum.

\section{Acknowledgements}

The authors would like to thank the school of MACE at the University of Manchester for the financial support. The first author would also like to thank Mr. Craig Hale and Mr. Ebrahim Erfani for their technical advice. The help and support of the administrative and technical staff of the University of Manchester are also greatly acknowledged.

\section{Appendix A. Response surface methodology}

In many RSM applications, the quadratic polynomial is employed as the prescribed model. The response surface is expressed as follows:

$y=b_{0}+\sum_{j} b_{j} x_{j}+\sum_{j} \sum_{i} b_{i j} x_{i} x_{j}+\epsilon$

where $Y$ is the response function, $x_{i}$ and $x_{j}$ are the design variables, and $b_{0}, b_{j}, b_{i j}$ are the unknown polynomial coefficients which are to be determined through regression analysis. $\epsilon$ represents the source of variability and uncertainty. In the literature [21], $\epsilon$ is a statistical error following normal distribution with mean zero and variance $\sigma^{2}$. The above equation can be written in its matrix notation as follows

$\mathbf{Y}=\mathbf{X b}+\epsilon$

where $\mathbf{Y}$ is the vector of response values and $\mathbf{X}$ is the following matrix

$\mathbf{X}=\left[\begin{array}{ccccc}1 & x_{11} & x_{12} & \ldots & x_{1 k} \\ 1 & x_{21} & x_{22} & \ldots & x_{2 k} \\ \vdots & \vdots & \vdots & \ddots & \vdots \\ 1 & x_{n 1} & x_{n 2} & \ldots & x_{n k}\end{array}\right]$

where $x_{i j}$ donate the $i$ th observation of level of variable $x_{j}$ [21].

A unique solution of Eq. (A.2), which is the least square estimator of $\mathbf{b}$ is

$\hat{\mathbf{b}}=\left(\mathbf{X}^{T} \mathbf{X}\right)^{-1} \mathbf{X Y}$.

Therefore, the fitted regression model is

$\hat{\mathbf{Y}}=\mathbf{X} \hat{\mathbf{b}}$.

\section{A.1. Design of experiments}

As discussed before, building an approximation model involves choosing the most revealing sample of data that represents the whole design space. DoE is a method for selecting the input parameter values as an appropriate sample at which a limited number of experiments are to be conducted for recording the response value.

In the literature, a variety of alternatives in DoE exist. These include Full Factorial designs, Fractional Factorial designs, PlackettBurman designs, Central-Composite designs, Taguchi designs, Doptimal designs, etc. [21]. All of these methods have their own pros and cons based upon the nature of the problem in the study. We investigate D-optimal experimental design, because of its favourable properties. It requires a fewer number of experimental runs and simulations compared to the other methodologies. In addition, it can be used for an irregular shaped design space.

\section{A.1.1. D-optimal experimental designs}

In D-optimal design a sample of experiments in a design space is chosen from a larger set of candidate points. The objective is to minimise the variance (uncertainty) in the estimated coefficients of the polynomial model (A.1). The minimisation of this variance is equivalent to maximisation of $\left|\mathbf{X}^{T} \mathbf{X}\right|$, where || is determinant operator [21]. In order to create D-optimal experimental designs, the optimisation methods are exploited. The most famous ones are genetic combinatorial optimisation and exchange coordinate algorithm.

\section{References}

[1] S. Agatonovic-Kustrin, M. Zecevic, L. Zivanovic, I.G. Tucker, Application of neural networks for response surface modeling in HPLC optimization, Analytica Chimica Acta 364 (1-3) (1998) 265-273.

[2] G.E.P. Box, N.R. Draper, Empirical Model-Building and Response Surfaces, Wiley Series in Probability and Mathematical Statistics: Applied Probability and Statistics, Wiley, 1987.

[3] Y.C. Cho, B. Jayaraman, F. Viana, R. Haftka, W. Shyy, Surrogate modelling for characterising the performance of a dielectric barrier discharge plasma actuator, International Journal of Computational Fluid Dynamics 24 (7) (2010) 281-301.

[4] C.L. Enloe, T.E. McLaughlin, R.D. VanDyken, K.D. Kachner, E.J. Jumper, T.C. Corke, Mechanisms and responses of a single dielectric barrier plasma actuator: Plasma morphology, AIAA Journal 42 (3) (2004) 589-594.

[5] C.L. Enloe, T.E. McLaughlin, R.D. VanDyken, K.D. Kachner, E.J. Jumper, T.C. Corke, M. Post, O. Haddad, Mechanisms and responses of a single dielectric barrier plasma actuator: Geometric effects, AIAA Journal 42 (3) (2004) 595-604.

[6] T. Erfani, S.V. Utyuzhnikov, Directed search domain: A method for even generation of the Pareto frontier in multiobjective optimization, Engineering Optimization 43 (5) (2011) 467-484.

[7] R. Erfani, C. Hale, K. Kontis, The influence of electrode configuration and dielectric temperature on plasma actuator performance, in: 49th AIAA Aerospace Sciences Meeting Including the New Horizons Forum and Aerospace Exposition, Orlando, 2011, Paper Number AIAA-2011-955.

[8] G.I. Font, Boundary layer control with atmospheric plasma discharges, AIAA Journal 44 (7) (2006) 1572-1578.

[9] M. Forte, J. Jolibois, J. Pons, E. Moreau, G. Touchard, M. Cazalens, Optimization of a dielectric barrier discharge actuator by stationary and non-stationary measurements of the induced flow velocity: Application to airflow control, Experiments in Fluids 43 (6) (2007) 917-928.

[10] M. Forte, J. Jolibois, F. Baudoin, E. Moreau, G. Touchard, M. Cazalens, Optimization of a dielectric barrier discharge actuator and non-stationary measurements of the induced flow velocity-Application to airflow control, in: 3rd AIAA Flow Control Conference, 2006, Paper Number AIAA-2006-2863.

[11] M. Gad-el Hak, Flow Control: Passive, Active, and Reactive Flow Management, Cambridge University Press, 2000.

[12] T. Goel, R.T. Haftka, W. Shyy, N.V. Queipo, Ensemble of surrogates, Structural and Multidisciplinary Optimization 33 (3) (2007) 199-216.

[13] S. Grundmann, C. Tropea, Delay of boundary-layer transition using plasma actuators, in: 46th AIAA Aerospace Sciences Meeting and Exhibit, 2008, Paper Number AIAA-2008-1369.

[14] C. Hale, R. Erfani, K. Kontis, Increasing the induced velocity of dielectric barrier discharge plasma actuators, in: CEAS 2009 European Air and Space Conference, 2009.

[15] C. Hale, R. Erfani, K. Kontis, Plasma actuators with multiple encapsulated electrodes to influence the induced velocity, in: 48th AIAA Aerospace Sciences Meeting Including the New Horizons Forum and Aerospace Exposition, 2010, Paper Number AIAA-2010-1223. 
[16] C. Hale, R. Erfani, K. Kontis, Plasma actuators with multiple encapsulated electrodes to influence the induced velocity: Further configurations, in: 40th Fluid Dynamics Conference and Exhibit, 2010, Paper Number AIAA-2010-5106.

[17] C. He, T.C. Corke, M.P. Patel, Plasma flaps and slats: An application of weakly ionized plasma actuators, Journal of Aircraft 46 (3) (2009) 864-873.

[18] J. Huang, T.C. Corke, F.O. Thomas, Plasma actuators for separation control of low-pressure turbine blades, AIAA Journal 44 (1) (2006) 51-57.

[19] J. Jacob, R. Rivir, C. Carter, J. Estevadeordal, Boundary layer flow control using AC discharge plasma actuators, in: AIAA 2nd Flow Control Meeting, Paper Number AIAA-2004-2128, 2004

[20] T. Jansson, L. Nilsson, M. Redhe, Using surrogate models and response surfaces in structural optimization-with application to crashworthiness design and sheet metal forming, Structural and Multidisciplinary Optimization 25 (2) (2003) 129-140.

[21] R.H. Myers, D.C. Montgomery, Response Surface Methodology, Allyn and Bacon, Boston, 1971.

[22] M. Negnevitsky, Artificial Intelligence: A Guide to Intelligent Systems, AddisonWesley/Longman, 2005.

[23] J. Nocedal, S.J. Wright, Numerical Optimization, Springer-Verlag, 1999.

[24] J. Pons, E. Moreau, G. Touchard, Asymmetric surface dielectric barrier discharge in air at atmospheric pressure: Electrical properties and induced airflow characteristics, Journal of Physics D: Applied Physics 38 (2005) 3635.

[25] C. Porter, T. McLaughlin, C. Enloe, G. Font, J. Roney, J. Baughn, Boundary layer control using a DBD plasma actuator, in: 45th AIAA Aerospace Sciences Meeting and Exhibit, Reno, 2007, Paper Number AIAA-2007-786.
[26] M.L. Post, T.C. Corke, Separation control on high angle of attack airfoil using plasma actuators, AIAA Journal 42 (11) (2004).

[27] M.L. Post, T.C. Corke, Separation control using plasma actuators: Dynamic stall vortex control on oscillating airfoil, AIAA Journal 44 (12) (2006) 3125.

[28] M. Raffel, C.E. Willert, J. Kompenhans, Particle Image Velocimetry: A Practical Guide, Springer-Verlag, 1998.

[29] J.R. Roth, X. Dai, Optimization of the aerodynamic plasma actuator as an electrohydrodynamic (EHD) electrical device, in: 44th AIAA Aerospace Sciences Meeting and Exhibit, Reno, 2006, Paper Number AIAA-2006-1203, pp. 9-12.

[30] J.R. Roth, D.M. Sherman, S.P. Wilkinson, Electrohydrodynamic flow control with a glow-discharge surface plasma, AIAA Journal 38 (7) (2000) 1166-1172.

[31] A. Samad, K.Y. Kim, T. Goel, R.T. Haftka, W. Shyy, Multiple surrogate modeling for axial compressor blade shape optimization, Journal of Propulsion and Power 24 (2) (2008) 302-310.

[32] A. Santhanakrishnan, J.D. Jacob, Y.B. Suzen, Flow control using plasma actuators and linear/annular plasma synthetic jet actuators, in: 3rd AIAA Flow Control Conference, 2006, Paper Number AIAA-2006-3033.

[33] T.W. Simpson, T.M. Mauery, J.J. Korte, F. Mistree, Kriging models for global approximation in simulation-based multidisciplinary design optimization, AIAA Journal 39 (12) (2001) 2233-2241.

[34] P. Versailles, V. Gingras-Gosselin, H.D. Vo, Impact of pressure and temperature on the performance of plasma actuators, AIAA Journal 48 (2010) 859863.

[35] B. Vladimir, H. Dan, G. Bernard, Wing design for a high-speed civil transport using a design of experiments methodology, 1996. 